\title{
The Routability of Multiprocessor Network Topologies in FPGAs
}

\author{
Manuel Saldaña, Lesley Shannon and Paul Chow \\ Dept. of Electrical and Computer Engineering \\ University of Toronto \\ Toronto, Ontario, Canada, M5S 3G4 \\ \{msaldana,lesley,pc\}@eecg.toronto.edu
}

\begin{abstract}
A fundamental difference between ASICs and FPGAs is that wires in ASICs are designed such that they match the requirements of a particular design. Wire parameters such as length, width, layout and the number of wires can be varied to implement a desired circuit. Conversely, in an FPGA, area is fixed and routing resources exist whether or not they are used, so the goal becomes implementing a circuit within the limits of available resources. The architecture for existing routing structures in FPGAs has evolved over time to suit the requirements of large, localized digital circuits. However, FPGAs now have the capacity to implement networks of such circuits, and system-level interconnection becomes a key element of the design process.

Following a standard design flow and using commercial tools, we investigate how this fundamental difference in resource usage affects the mapping of various network topologies to a modern FPGA routing structure. By exploring the routability of different multiprocessor network topologies with 8, 16 and 32 nodes on a single FPGA, we show that the difference between resource utilization of a ring, star, hypercube and mesh topologies is not significant up to 32 nodes. We also show that a fully-connected network can be implemented with at least 16 nodes, but with 32 nodes it exceeds the routing resources available on the FPGA. We also derive a cost metric that helps to estimate the impact of the topology selection based on the number of nodes.
\end{abstract}

\section{Categories and Subject Descriptors}

C.1.2 [Processor Architectures]: Multiple Data Stream Architectures (Multiprocessors); D.0 [Computer Systems Organization]: General

\section{General Terms}

Design

Permission to make digital or hard copies of all or part of this work for personal or classroom use is granted without fee provided that copies are not made or distributed for profit or commercial advantage and that copies bear this notice and the full citation on the first page. To copy otherwise, to republish, to post on servers or to redistribute to lists, requires prior specific permission and/or a fee.

SLIP'06, March 4-5, 2006, Munich, Germany.

Copyright 2006 ACM 1-59593-255-0/06/0003 ...\$5.00.

\section{Keywords}

Multiprocessor, FPGA, Network-on-Chip, Topology, Interconnect

\section{INTRODUCTION}

With the growing complexity of System-on-Chip (SoC) circuits, more sophisticated communication schemes are required to connect the increasing number and variety of intellectual property (IP) blocks. Approaches like AMBA [1], CoreConnect [2], WISHBONE [3] and SiliconBackplane [4] follow a shared bus scheme that works well for MasterSlave communication patterns, where there are peripherals (slaves) that wait for data to be received or requested from a more complex processing IP (master). When there are several masters (e.g., processors) in the system, synchronization, data interchange and $\mathrm{I} / \mathrm{O}$ may saturate the bus, and contention will slow down data transfers.

The Network-on-Chip (NoC) $[5,6]$ provides a possible solution for this problem by creating a scalable interconnection scheme. The concept uses a set of buses connected to routers or switches that interchange packets, much in the same way as traditional computer networks or multiprocessor machines do. Consequently, NoC approaches have design parameters and properties similar to traditional networks. One of these parameters is the topology, which defines the interconnection pattern between the routers and switches.

Multiple topologies have been studied for NoCs on ASICs [7] [8]. A popular choice is the mesh $[6,9]$ because it provides structure, better control over electrical characteristics, has an easy packet routing algorithm. These advantages are clear for ASICs, but not necessarily for FPGAs [10]. The electrical characteristics of the FPGA are solved by the chip vendor, not by the user. As for structure it is perhaps intuitive to use a mesh topology in FPGAs since the reconfigurable fabric layout is in the form of a mesh. However, the placement and routing of components on an FPGA will not typically result in a symmetric, well-organized structured layout that resembles a mesh. Furthermore, manually restricting the placement of components or routing of nets may lead to inefficient resource utilization for the logic that is not part of the network. Finally, there are other topologies like hypercube or torus networks, or even tree topologies that also have simple routing algorithms.

In this paper, we compare the routability of point-to-point network topologies on FPGAs by measuring the impact of each topology on a soft multiprocessor system implemented on modern commercial FPGAs. We do this by measur- 
ing the logic utilization, logic distribution (area), maximum clock frequency, number of nets, and the place and route time for five different network topologies. We also derive a cost metric to try to extract trends for larger systems.

The rest of this paper is organized as follows. Section 2 provides some background about research on NoCs. Section 3 describes the topologies implemented and gives a brief description of the block used as the network nodes, which we call the computing node. Section 4 describes the implementation platform, and how the systems are generated. Section 5 presents the results obtained for the baseline system and Section 6 explores the chip area required for each topology. Section 7 shows the highest frequency that each system could achieve. Section 8 presents a metric we propose to evaluate the topologies and Section 9 provides some conclusions.

\section{RELATED WORK}

In this section we present examples of typical research on NoCs, and how it relates to this study. In Brebner and Levi [10] discuss NoC implementations on FPGAs, but their focus is on the issues of using packet switching on a mesh topology in the FPGA and on implementing crossbar switches in the routing structure of the FPGA. Most NoC work assumes ASIC implementations and there are numerous studies including work on mesh topologies [6][9] and fat trees [7]. Other studies on NoCs are done using registertransfer-level simulations [7] and simulation models [11], but they do not show the implementation side of the NoC. Instead, we focus on the interaction between the network topologies and how well they can be mapped to a fixed FPGA routing fabric. We create actual implementations by performing synthesis, mapping, placement and routing for real FPGAs using commercial tools.

Research has been done on synthesizing application-specific network topologies [12]. A more general study on the routability of different topologies would require the ability to generate arbitrary interconnection patterns. In our work, we created a design flow and tools to automatically generate multiprocessor systems using a set of well known topologies.

Based on the philosophy of routing packets, not wires [9, 13], NoC architectures have been proposed as packetswitching networks, with the network interface itself being the focus of much of the research. In this paper, we use a simple network interface, more similar to a network hub than a switch as it does not provide packet forwarding. Packets can only be sent to, and received from nearest-neighbor nodes. This makes the network interface extremely simple, but it is sufficient for our purposes as the focus of this work is on the routability of various topologies, not on the switching element architecture.

\section{EXPERIMENTAL ENVIRONMENT}

The actual processor and network interface used are not the critical elements in this study. What is required is to create circuits that force particular routing patterns between the computing nodes to see how the implementation resources of these circuits on the FPGAs varies as the patterns, i.e., topologies, are changed. We try five different topologies and three different system sizes $(8,16$ and 32 nodes) on five different FPGAs with enough resources to implement such systems.
Table 1: Characteristics of the topologies studied

\begin{tabular}{|l|c|c|c|c|c|}
\hline Topology & Diameter & $\begin{array}{c}\text { Link } \\
\text { Complexity }\end{array}$ & Degree & Regular & $\begin{array}{c}\text { Bisection } \\
\text { Width }\end{array}$ \\
\hline ring & $\frac{n}{2}$ & $n$ & 2 & yes & 2 \\
\hline star & 2 & $n-1$ & $1, n-1$ & no & 1 \\
\hline $\begin{array}{l}\text { square } \\
\text { mesh }\end{array}$ & $2\left(n^{1 / 2}-1\right)$ & $2\left(n-n^{1 / 2}\right)$ & $2,3,4$ & no & $2 \sqrt{n}$ \\
\hline hypercube & $\log _{2} n$ & $\frac{n \log _{2} n}{2}$ & $\log _{2} n$ & yes & $\frac{n}{2}$ \\
\hline $\begin{array}{l}\text { fully } \\
\text { connected }\end{array}$ & 1 & $\frac{n(n-1)}{2}$ & $n-1$ & yes & $\frac{n^{2}}{4}$ \\
\hline \hline
\end{tabular}

In this section we describe the Network-on-Chip we used to perform the experiments, which are explained later in this paper.

\subsection{Network Topologies}

Networks can be classified into two categories. Static networks consist of point-to-point, fixed connections between processors, and dynamic networks which have active elements, such as switches, that can change the connectivity pattern in the system according to a protocol. In an FPGA, the network can be dynamically reconfigured to adapt to communication patterns by utilizing the reconfigurability [14] of the FPGA.

In this paper, we focus on static message passing networks. The ring, star, mesh, hypercube and fully-connected topologies are selected as a representative sample, ranging from the simplest ring topology to the routing-intensive fullyconnected system.

Network topologies can be characterized by a number of properties: node degree, diameter, link complexity, bisection width and regularity [15]. Node degree is the number of links from a node to its nearest neighbors. Diameter is the maximum distance between two nodes. Link complexity is the number of links the topology requires. A network is deemed to be regular when all the nodes have the same degree. Bisection width is the number of links that must be cut when the network is divided into two equal set of nodes. Table 1 shows a summary of these characteristics for each of the topologies used in this paper.

The characteristics of the network topology define the network interface of a node. For example, the four-dimensional hypercube is a regular topology, with all nodes having a degree of four. This means that this topology requires a single network interface type, each with four ports, i.e., four communication links. The network interface is used to communicate with other nodes in the network. The maximum distance (diameter) is four, which means that data going through the network may require redirection or routing at intermediate nodes and travel on up to four links. The link complexity is 32 , which is the total number of point-to-point links that the overall system will have. In contrast, a 16node mesh has a total of 24 links in the system, but it is not a regular topology, requiring three different versions of the network interface. Inner nodes require an interface with four ports, perimeter nodes require one with three ports and corner nodes use a two-port interface.

Figure 1 shows examples of systems with different numbers of nodes and topologies that are implemented to carry out our experiments. Every topology can be seen as a graph that is made of edges (links) and vertices (computing nodes). In our implementations, the links are 64 bits wide 
(i.e. Channel_width of 64 bits) with 32 bits used for transmission and 32 bits used for reception, making it a full-duplex communication system. The links also include control lines used by the network interface.
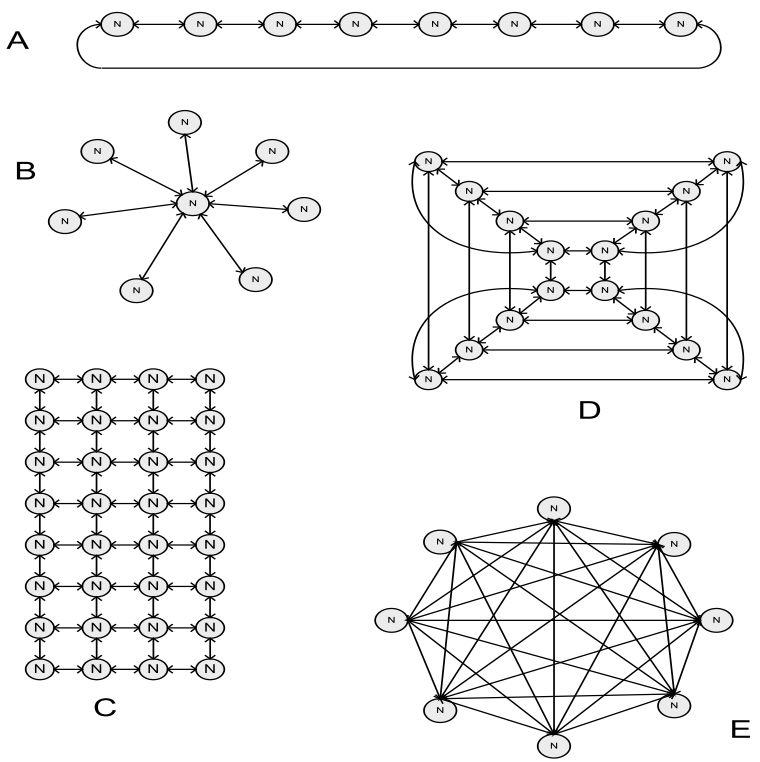

Figure 1: A) 8-node ring, B) 8-node star, C) 32node mesh, D) 16-node hypercube, and E) 8-node fully-connected topology

\subsection{Computing Node}

The computing nodes in Figure 1 consist of a computing element and a network interface module. Figure 2 shows the structure of a computing node. The master computing node of the system is configured to communicate with the external world using a UART attached to the peripheral bus shown inside the dashed box of Figure 2. The rest of the nodes have no peripheral bus.

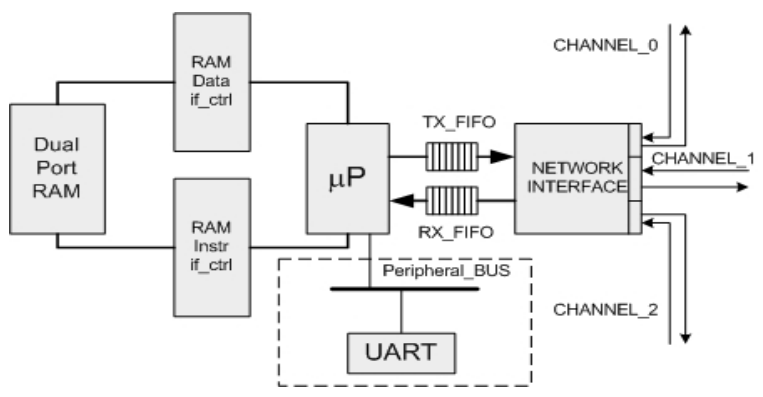

Figure 2: The computing node

We use a Harvard architecture soft core processor as the computing element so that data memory and program memory are accessed by independent memory buses. The communication between the computing element and the network interface is achieved by using two 32-bit wide FIFOs: one for transmission and one for reception.

The network interface module is an extremely simple block that has two sides. It interfaces to the network with several links (channels) according to the degree of the node.
On the other side, two FIFOS are used as message buffers to the processor.

The network interface is basically a hub that broadcasts the data to the neighbors on transmission, and it filters out the data from the neighbors on reception. It is effectively a FIFO multiplexer that is controlled by the destination field in the packet header. If the destination value matches the processor's ID number, then the packet passes through the hub to the processor attached to the hub. Again, this interface is simple, but is enough for the purpose of this research, since we are interested in the connectivity pattern.

Implementing a single version of the network interface would not provide a good measure of the difference in logic utilization between the various topologies because the topologies requiring nodes of lesser degree should use less logic. It is likely that the optimizer in the synthesis tool would remove the unused ports, still allowing the study to be performed, but we chose to actually implement the different node degrees required to be certain that only the necessary logic was included.

The size of the remaining logic in the computing node is independent of the node degree i.e., the logic in the processors, the FIFOs, the memory controllers and the UART are independent of the topology selection.

\section{IMPLEMENTATION PLATFORM}

To build the net list, map the design, place it and route it, we use the Xilinx [16] EDK tools version 7.1i in combination with the Xilinx XST synthesis tool. To visualize the placement of the systems, we use the Xilinx FPGA Floorplanning tool. The network interface is developed in VHDL and simulated using ModelSim version 6.0b [17]. The routed nets are counted with the help of the Xilinx FPGA Editor. For Section 7, we use the Xilinx Xplorer utility to try to meet the timing constraints. All the experiments are executed on an IBM workstation with a Pentium 4 processor running at $2.8 \mathrm{GHz}$ with Hyperthreading enabled and $2 \mathrm{~GB}$ of memory.

Our multiprocessor systems use the Xilinx MicroBlaze soft-processor core [16] as the computing element. The computing element connects to the network interface module through two Fast Simplex Links (FSL), a Xilinx core that is a unidirectional, point-to-point communication bus implemented as a FIFO.

We use a variety of Xilinx chips to implement the designs: the Virtex2 XC2V2000, and the Virtex4 XC4VLX25, XC4VLX40, XC4VLX60 and XC4VLX200. The LX version of the Virtex4 family only has Block RAM (BRAM) and DSP hard cores in addition to the FPGA fabric. They do not have PowerPC processors or Multi-gigabit Transceivers (MGTs). This provides a more homogeneous architecture that facilitates area comparisons.

The hard multiplier option for the MicroBlaze is disabled to minimize the impact of hard core blocks that may influence or limit the placement and routing. The BRAM are hard core blocks that also affect placement and routing, but they are essential for the MicroBlaze system to synthesize so they have not been eliminated.

A 32-node, fully-connected system requires 1056 links to be specified, and doing this manually is time consuming and error prone. Instead, we developed a set of tools that take a high-level description of the system that specifies the topology type, the number of nodes, the number of total links and the number of links per node, and they generate the 
files required by EDK.

The number of nodes, for all the topologies, is chosen based on the limitation of the hypercube to $2^{d}$ nodes, where $d$ is the dimension. For $d=3,4$ and 5 we have 8, 16 and 32 nodes, respectively.

\section{BASELINE SYSTEM}

The main objective of this experiment is to measure the logic and routing resources required for each of the topologies. The timing constraints are chosen to be realistic, but not aggressive, so that the place and route times are not excessive. The 8 and 16-node systems are specified to run at $150 \mathrm{MHz}$ and the 32-node systems are specified to run at $133 \mathrm{MHz}$ to account for the slower speed grade of the XC4VLX200 chip that is used for those systems.

The logic resource usage is measured in terms of the total number of LUTs required for a design and the number of LUTs related to only the interconnection network, i.e., those used to implement the network interface modules. The logic resources needed to implement the network are estimated by first synthesizing the network interface modules as standalone blocks to determine the number of LUTs required. These numbers are then used to estimate the usage of the entire network. For example, the 8-node star topology requires one 7-port network interface, which uses 345 LUTs, and seven 2-port network interfaces, which need 111 LUTs each. The total number of LUTs required by the network is $345+(7 \times 111)=1122$ LUTs. Note that this is only an estimate as the values reported by synthesizing the stand-alone block level may change at the system level due to optimizations that may occur. The register (flip flop) utilization is found by using the same method as used for finding the logic resource utilization.

The routing resource utilization is measured in terms of the total number of nets in the design and the number of nets used to implement only the network links and network interfaces. The counting of nets is done by using the Xilinx FPGA Editor, which allows the user to filter out net names. The number of nets attributed to the network is found by counting the number of nets related to all the network interface modules in the design. This includes all nets that are used in the network interface module as well as the nets in the network topology itself. Including the nets in the network interface module is reasonable because more complex topologies use more complex network interfaces that also consume FPGA routing resources.

\subsection{Results}

Figure 3 shows a histogram of the number of LUTs needed to implement the complete systems, including the MicroBlaze, FSLs, memory interface controllers, switches, UART, and OPB bus. As expected, the system with the fullyconnected network has the highest logic utilization, and as the system size increases, the difference with respect to the other topologies gets more pronounced because of the $O\left(n^{2}\right)$ growth in size. The difference is most significant with the 32 -node system, which requires over twice the logic of the other systems. For the other topologies, the maximum difference in LUT usage amongst the topologies at the same node size ranges from about $5 \%$ in the 8 -node systems to about $11 \%$ in the 32 -node systems.

A more detailed view of the logic resources can be seen in Table 2. The Logic Utiliz. column is the total number of

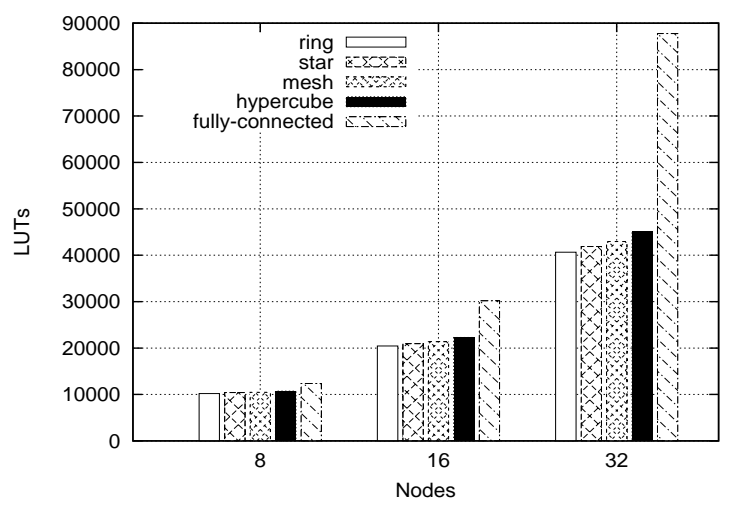

Figure 3: Logic utilization of systems

Table 2: Logic and register resources used by each system

\begin{tabular}{|l|c|l|r|r|r|r|r|}
\hline Topology & Nodes & $\begin{array}{c}\text { Logic } \\
\text { Utiliz. } \\
\text { LUTs })\end{array}$ & $\begin{array}{r}\text { Logic } \\
\text { Incr. } \\
(\%)\end{array}$ & $\begin{array}{r}\text { Logic } \\
\text { Ovrhd. } \\
(\%)\end{array}$ & $\begin{array}{r}\text { Total } \\
\text { Reg. }\end{array}$ & $\begin{array}{r}\text { Reg. } \\
\text { Incr. } \\
(\%)\end{array}$ & $\begin{array}{r}\text { Reg. } \\
\text { Ovrhd. } \\
(\%)\end{array}$ \\
\hline ring & 8 & 10197 & 0.0 & 8.7 & 2637 & 0.0 & 11.2 \\
\hline star & 8 & 10393 & 1.9 & 10.8 & 2642 & 0.2 & 11.6 \\
\hline mesh & 8 & 10470 & 2.7 & 10.7 & 2641 & 0.2 & 11.4 \\
\hline hypercube & 8 & 10701 & 4.9 & 12.6 & 2645 & 0.3 & 11.5 \\
\hline fully con. & 8 & 12376 & 21.4 & 22.3 & 2762 & 4.7 & 13.9 \\
\hline \hline ring & 16 & 20448 & 0.00 & 8.7 & 5186 & 0.0 & 11.4 \\
\hline star & 16 & 20936 & 2.4 & 9.6 & 5190 & 0.1 & 11.8 \\
\hline mesh & 16 & 21360 & 4.5 & 12.6 & 5202 & 0.3 & 11.7 \\
\hline hypercube & 16 & 22272 & 8.9 & 16.2 & 5218 & 0.6 & 12.0 \\
\hline fully con. & 16 & 30176 & 47.6 & 38.1 & 5490 & 5.9 & 16.3 \\
\hline \hline ring & 32 & 40648 & 0.00 & 8.7 & 10209 & 0.0 & 11.6 \\
\hline star & 32 & 41880 & 3.0 & 9.0 & 10214 & 0.1 & 11.9 \\
\hline mesh & 32 & 42936 & 5.6 & 13.6 & 10250 & 0.4 & 11.9 \\
\hline hypercube & 32 & 45104 & 11.0 & 17.9 & 10306 & 0.9 & 12.4 \\
\hline fully con. & 32 & 87760 & 115.9 & 57.8 & 11330 & 11.0 & 20.3 \\
\hline \hline
\end{tabular}

LUTs used for each design and these are the values shown in Figure 3. Since the ring has the simplest routing topology, it is used as the baseline for comparisons with the rest of the topologies.

Column Logic Incr. shows the increase in the number of LUTs for each topology relative to the ring topology. For example, the fully-connected topology requires $21.4 \%$ more LUTs than the ring for the 8-node system. In contrast, the Logic Ovrhd column shows the number of LUTs used for the network interfaces as a fraction of the total LUTs required for the complete system. It is calculated as (total number of LUTs for network interfaces)/(Total LUTs in the system). As expected, the ring topology has the lowest overhead for all node sizes and the fully-connected system overhead increases very quickly as the number of nodes increases.

Table 2 also shows the corresponding results for the register (flip flop) utilization of the various topologies. The trends mimic the logic utilization data, but the variation is smaller because the number of registers in the network interface module is small and because it is the only component that is changing in size.

The routing resource usage of each system is presented in Table 3. The Routing Utiliz. column is the total number 
of nets used in the design. In general, the routing resource utilization follows a similar pattern to the logic resource utilization across the systems. The fully-connected system requires the most nets, as expected. It should also be noted that the 32-node, fully-connected topology design could be placed but not completely routed, leaving 56 unrouted nets.

Column Routing Increase presents the difference in routing resources relative to the ring topology. It can be seen that the greatest increase in routing for the ring, star, mesh and hypercube topologies occurs for the 32-node hypercube system with only a $10.6 \%$ increase relative to the 32 -node ring system. This reflects the $O(n \log n)$ link complexity of the hypercube as compared to the $O(n)$ link complexity for the ring, star, and mesh topologies.

The Routing Ovrhd column is calculated as the total number of nets for all the network interfaces divided by the total number of nets in the entire system. A visual representation of how each network topology contributes to the global number of nets can be seen in Figure 4. From this figure it can be seen that the ring topology overhead is practically independent of the system size at about $6 \%$ of the total nets for the 8, 16 and 32-node systems. The star and mesh topologies increase slowly to a maximum of about $11 \%$ of the total nets for the 32-node system. The hypercube adds about $15 \%$ overhead to the global routing in the 32 -node system. The fully-connected topology starts at $20 \%$ overhead for an 8-node system, and grows to around $55 \%$ of the total routing for the 32-node topology, which actually fails to completely route. The other topologies have much lower routing overhead and will likely be able to expand to 64node or 128-node systems, assuming large enough FPGAs exist.

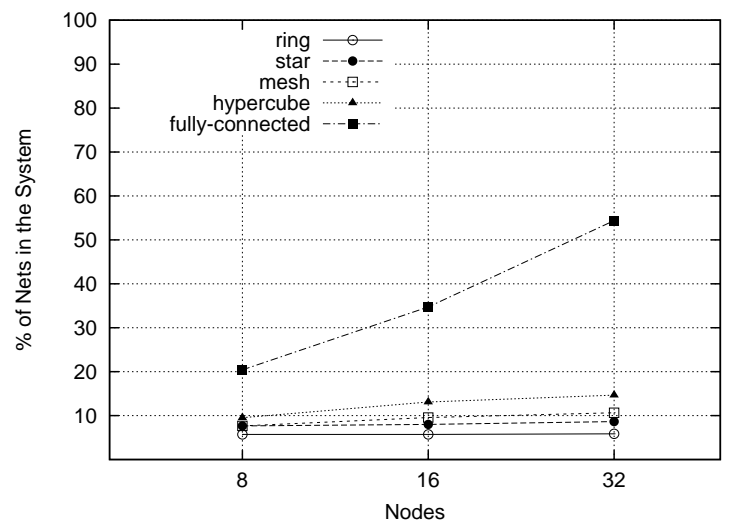

Figure 4: Topology impact on global routing

The place and route time data varies considerably because of how the place and route algorithms work and factors that impact the workstation performance. For the ring, star, mesh and hypercube topologies, the average times to place and route are approximately the same for a fixed number of nodes.

For the 8, 16 and 32-node systems the average times are 12 min., 30 min. and 4 hours 48 min., respectively. The fully-connected topology exhibits an exponentially growing time of $15 \mathrm{~min}$. for the 8-node system, 12 hours for the 16node system, and remained unroutable after 3 days for the 32-node system.

Table 3 also shows the clock frequency (freq.) achieved
Table 3: Routing resources used by each system \begin{tabular}{|l|l|l|l|}
\hline Topology|NodesRouting & Routing & Routing & freq. \\
\hline
\end{tabular}

\begin{tabular}{|l|c|r|r|r|r|r|}
\hline ring & 8 & 10744 & 0.0 & 5.7 & 150 & 150 \\
\hline star & 8 & 10956 & 2.0 & 7.6 & 151 & 150 \\
\hline mesh & 8 & 11021 & 2.6 & 7.7 & 151 & 150 \\
\hline hyp.cube & 8 & 11256 & 4.8 & 9.5 & 152 & 150 \\
\hline fully con. & 8 & 13045 & 21.4 & 20.4 & 150 & 150 \\
\hline \hline ring & 16 & 21501 & 0.0 & 5.7 & 152 & 150 \\
\hline star & 16 & 22013 & 2.4 & 8.0 & 150 & 150 \\
\hline mesh & 16 & 22429 & 4.3 & 9.6 & 151 & 150 \\
\hline hyp.cube & 16 & 23357 & 8.6 & 13.1 & 151 & 150 \\
\hline fully con. & 16 & 31373 & 45.9 & 34.7 & 128 & 150 \\
\hline \hline ring & 32 & 42618 & 0.0 & 5.8 & 133 & 133 \\
\hline star & 32 & 43888 & 3.0 & 8.6 & 100 & 133 \\
\hline mesh & 32 & 44945 & 5.5 & 10.6 & 132 & 133 \\
\hline hyp.cube & 32 & 47136 & 10.6 & 14.7 & 133 & 133 \\
\hline fully con. & 32 & 90016 & 111.2 & 54.4 & Fail & 133 \\
\hline \hline
\end{tabular}

for each of the systems. Of the 8 and 16-node systems, only the fully-connected, 16-node system is not able to meet the $150 \mathrm{MHz}$ requirement, achieving only $128 \mathrm{MHz}$. With the 32-node systems, the target is $133 \mathrm{MHz}$, but this is not achieved by the star or the fully-connected network. The star incurs congestion at the central node, which affects the timing, and the fully-connected system requires too many wires. The placement and routing efforts were set to high, but no time was spent to try and push the tools to improve the results that did not meet the targets.

\section{AREA REQUIREMENTS}

For the previous experiments, LUT and flip flop counts are used as the reference metrics for logic resource utilization. However, for this experiment the number of slices is used to measure area usage. In the Xilinx architecture, each slice contains two LUTs. A design requires a certain number of LUTs and flip flops, and depending on how well the packing algorithm performs, the design will require more or less slices. Moreover, the place and route tools may not be able to utilize the two LUTs in every slice because of routing constraints and timing requirements. The number of slices better reflects the actual chip area required to implement the design. Also, the area constraints used by the Xilinx tools are specified in terms of slices.

For this experiment, the Minimum Area Required is defined as the smallest number of slices needed for the design to place and route successfully. It is determined by reducing, or compressing, the area used by the design until just before it fails to place and route and counting the number of slices in the compressed region at that point. This gives a measure of how efficiently the design can use the resources when the resources are close to being fully utilized, which models the effect of trying to implement a design on a chip that is close to full capacity.

The area compression is done using area constraints in the User Constraints File, i.e., the .ucf file. The constrained area is described by giving the coordinates of the bottomleft and top-right slice positions that define a rectangular area in the FPGA. The origin is fixed to $X 0 Y 0$ and the 
second $X$ coordinate is fixed at the maximum value allowed by the specific chip, i.e., the width of the FPGA fabric. The variable is the second $Y$ coordinate. Decreasing the $Y$ coordinate compresses the area available for the design and an iterative process of changing this coordinate is used to find the Minimum Area Required.

We define Area Utilization as the ratio of the number of slices actually used by the design to the total number of slices in the area available to the design. The ideal area utilization is to use $100 \%$ of the slices available to the design.

In this experiment, the 8-node systems using the five topologies are compared using three different chips. This experiment also requires a time-intensive, iterative search to find the Minimum Area Required so the 16 and 32-node systems are not studied.

The fact that the MicroBlaze soft processor needs internal memory (BRAM) to store the code and data required a slight modification to our methodology. BRAMs are hard core macros that have fixed positions within the FPGA fabric, and they are organized as blocks of 18 Kbits each, distributed along different columns in the array. A problem may arise if the restricted area does not have enough BRAMs for the design. In that case, the map program will issue an error about the lack of resources or resources being over mapped. For that reason, the BRAM blocks are not constrained in our methodology. This implies that nets connected to the BRAMs may be outside of the restricted area. However, each MicroBlaze uses only 8 KB of BRAM, which is only four BRAM blocks. In all of the designs, the number of BRAMs outside the restricted area is small and the number of nets involved are not significant compared to the total number of nets in the design.

To ensure that the resulting maximum clock frequency is realistic, the timing requirement is set to $150 \mathrm{MHz}$, but a successful place and route in this experiment does not require that timing be met, only that it comes within about $20 \mathrm{MHz}$ to ensure a respectable clock speed. The important requirement is that there are sufficient resources to complete a place and route. All of the Virtex 4 designs are able to achieve $150 \mathrm{MHz}$ using high effort. The Virtex 2 device, being in a slower technology only has to meet $133 \mathrm{MHz}$ as the timing constraint. The speeds achieved are $133 \mathrm{MHz}$ for the ring, star and hypercube, $125 \mathrm{MHz}$ for the mesh and $117 \mathrm{MHz}$ for the fully interconnected system. These results are also achieved using high effort.

\subsection{Results}

Table 4 shows the minimum number of slices required (Min. Area Req'd) and the area utilization (Area Utiliz.) for the 8-node systems. Observe that a higher area utilization can be achieved in the Virtex 2 chip compared to the Virtex 4 chips and the XC4VLX40 achieves slightly higher utilization than the XC4VLX25.

Within the same chip, the fully-connected topology achieves equal or higher area utilization ratios than the rest of the topologies, even if the other topologies require fewer resources than the fully-connected one. This occurs because the fully-connected has more logic to place in the empty spaces caused by MicroBlaze macros that have holes of unused slices in them due to the use of relational placement directives. These directives force certain parts of the logic into fixed relative positions, which results in unused slices within the bounds of the macro. In this case, the fully-connected
Table 4: Area utilization for the 8-node systems using different chips

\begin{tabular}{|c|c|c|c|c|c|c|}
\hline Topology & \multicolumn{2}{|c|}{ XC2V2000 } & \multicolumn{2}{c|}{ XC4VLX25 } & \multicolumn{2}{c|}{ XC4VLX40 } \\
\hline & $\begin{array}{c}\text { Min. Area } \\
\text { Req'd } \\
\text { (slices) }\end{array}$ & $\begin{array}{c}\text { Area } \\
\text { Utiliz. } \\
(\%)\end{array}$ & $\begin{array}{c}\text { Min. Area } \\
\text { Req'd } \\
\text { (slices })\end{array}$ & $\begin{array}{c}\text { Area } \\
\text { Utiliz. } \\
(\%)\end{array}$ & $\begin{array}{c}\text { Min. Area } \\
\text { Req'd } \\
\text { (slices) }\end{array}$ & $\begin{array}{c}\text { Area } \\
\text { Utiliz. } \\
(\%)\end{array}$ \\
\hline ring & 5376 & 92.5 & 9352 & 62.1 & 8208 & 70.7 \\
\hline star & 5568 & 90.8 & 8736 & 67.5 & 8640 & 68.2 \\
\hline mesh & 5376 & 94.4 & 9240 & 64.0 & 8280 & 73.5 \\
\hline hypercube & 6336 & 81.9 & 8568 & 70.4 & 8280 & 72.9 \\
\hline fully con. & 6528 & 92.5 & 9688 & 71.2 & 8208 & 84.1 \\
\hline \hline Average & 5836 & 90.4 & 9116 & 67.0 & 8323 & 73.9 \\
\hline \hline
\end{tabular}

topology can make more effective use of the empty spaces because it has more logic to place.

Table 4 also shows that the average Min. Area Req'd slices varies quite significantly across the different chips. A part of the explanation is due to an architectural change to the Configurable Logic Block (CLB) between the Virtex 2 and the Virtex 4 families. The effect of the change is that the implementation of the Microblaze in Virtex 4 uses 160 more LUTs than in a Virtex 2. This difference explains most of the differences in LUT requirements between the two FPGA families, which accounts for part of the difference in slice counts. The other important reason for the differences in area required arises because of the relative width of the MicroBlaze macro to the width of the FPGA fabric. For the same amount of logic, Virtex 2's fabric is wider than Virtex 4's fabric.

\section{MAXIMUM FREQUENCY}

The goal of this experiment is to determine the maximum frequency achievable by the different topologies. This is done by using the Xilinx Xplorer utility, which is a Perl script that runs the map, and place and route tools up to six times. Each run uses a different combination of command line options that enable the tools to try various combinations of optimizations to meet the timing constraints. These options set the mapping effort level to high, place and route effort levels to high, try different seeds, enable timing-driven packing, global optimizations, register duplication and alternative algorithms for the mapping tool. The specified frequency is $180 \mathrm{MHz}$ because it is the frequency at which the MicroBlaze synthesizes, being the slowest IP block in the system.

The fastest Speed Grade available for each chip is used. The XC4VLX200 is the largest part and it has a maximum speed grade of 11, which is not as fast as what is available for the other chips we use. The method for finding the maximum frequency is an iterative process and it takes a great amount of time to map, place and route all of the systems. Instead, the full set of 16-node systems in combination with one example from each of the 8 and 32-node systems are selected for this study. The fully-connected, 8-node network is chosen because it is the most challenging of the 8-node systems. The remaining 8 -node networks should be easier than the corresponding 16-node networks. The 32-node ring system is selected because it is the easiest topology of the set of 32-node networks. 
Table 5: Results from running Xilinx Xplorer

\begin{tabular}{|l|c|c|c|c|c|}
\hline Topology & Nodes & $\begin{array}{c}\text { Max } \\
\text { Freq. } \\
(\mathrm{MHz})\end{array}$ & $\begin{array}{c}\text { Speed } \\
\text { Grade }\end{array}$ & $\begin{array}{c}\text { Best } \\
\text { Run }\end{array}$ & $\begin{array}{c}\text { Total } \\
\text { Runs }\end{array}$ \\
\hline fully con. & 8 & 170 & 12 & 2 & 6 \\
\hline \hline ring & 16 & 180 & 12 & 1 & 1 \\
\hline star & 16 & 180 & 12 & 4 & 4 \\
\hline mesh & 16 & 180 & 12 & 2 & 2 \\
\hline hypercube & 16 & 180 & 12 & 2 & 2 \\
\hline fully con. & 16 & 126 & 12 & 5 & 6 \\
\hline \hline ring & 32 & 123 & 11 & 5 & 6 \\
\hline \hline
\end{tabular}

\subsection{Results}

Table 5 shows the results of executing the Xilinx Xplorer utility. The maximum frequency achieved is shown in Column Max Freq.. The 8-node, fully-connected system fails to meet the $180 \mathrm{MHz}$ constraint. An examination of the longest critical paths shows that $88 \%$ of the delay is attributed to routing delay suggesting that routing is the main factor for not meeting timing.

Most of the 16-node systems meet the target frequency of $180 \mathrm{MHz}$. Only the 16-node, fully-connected system is unable to meet the timing constraints. Examination of the timing report shows that for the longest critical paths, $72 \%$ of the delay is due to routing.

Interestingly, the 32-node ring is able to achieve $133 \mathrm{MHz}$ in the previous experiments with about $50 \%$ of the critical path delay in the routing, but when the constraint is changed to $180 \mathrm{MHz}$ only $123 \mathrm{MHz}$ is achieved, with $72 \%$ of the critical path delay being in routing.

The Best Run is the iteration number in which the maximum frequency is obtained. The column Total Runs shows the total number of iterations for which the script invoked the map, place and route tools. The maximum value is 6 , but it is less if the timing constraint is met in fewer iterations. In the case of the ring with 16 nodes, the topology is so simple that the maximum frequency is achieved by the first run. The star needed more runs indicating the tools have to work harder due to the congestion around the central node. Adding a few more nodes to the star will likely cause it to fail to meet the $180 \mathrm{MHz}$ target. The fully-connected system requires six runs and still cannot meet the timing constraint, getting the best result in iteration number 5 . A similar situation happens with the 32-node ring system.

The size of the MicroBlaze macros dictates the overall placement and will not change significantly for the different topologies. Therefore, the placement of the computing nodes is not the important factor for failing to meet timing constraints. The only other factor would be the routing. In this experiment, it is observed that routing delay is the major component of the critical paths of the star and the fully-connected 8 and 16-node topologies, which is indicative of routing being the problem. From what we know about the star and fully-connected topologies, routing congestion becomes the problem for large node counts, which supports the observation of routing delay being the major component of the critical paths.

\section{A COST METRIC}

There are many factors that affect the routability of a network and to expose possible interesting trends, we propose

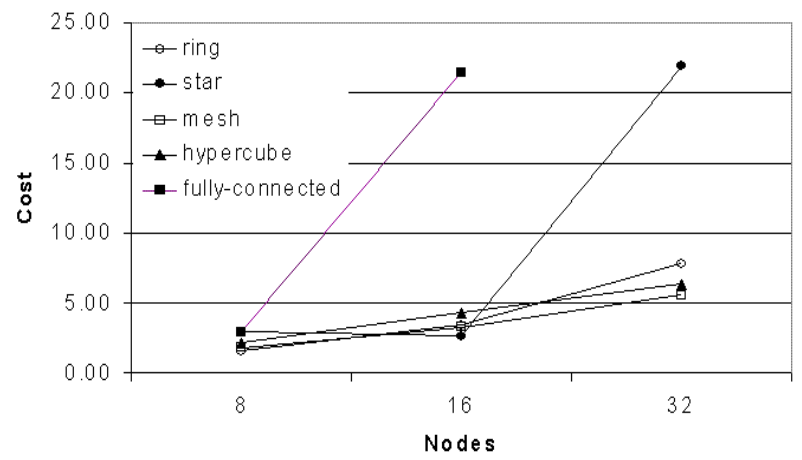

Figure 5: Cost metric trends

the following cost metric $(C M)$ to minimize:

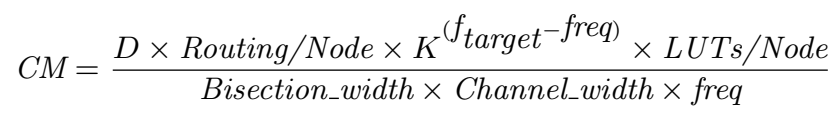

where $D$ is the network diameter computed using the appropriate expression in Table 1. Recall that a small diameter indicates fewer links need to be traversed in the worst case; therefore a lower latency. The Routing/Node and LUTs/Node are the number of nets per node and LUTs per node attributed to the network, respectively. They are computed as:

$$
\begin{gathered}
\text { Routing } / \text { Node }=\frac{\text { Routing Utiliz. } \times \text { Routing Ovrhd (\%) }}{\text { Nodes }} \\
\text { LUTs } / \text { Node }=\frac{\text { Logic Utiliz. } \times \text { Logic Ovrhd (\%) }}{\text { Nodes }}
\end{gathered}
$$

using the entries in Table 2 and Table 3 . The $K^{\left(f_{\text {target }}{ }^{-f r e q}\right)}$ value is a weighting performance factor used to bias the cost metric such that topologies that operate at less than the desired clock frequency are penalized, whereas topologies that achieve more than the desired frequency are rewarded. The $f_{\text {target }}$ is the target clock and freq is the actual frequency as reported in Table 3 . We found, by trial and error, that a base factor of $K=1.05$ is enough to cause the systems that did not meet timing to have the highest costs.

If we assume a data word is transmitted every clock cycle, then the denominator represents the bisection bandwidth, which is defined as the Bisection Width (Table 1) times the bit rate $($ Channel_width $\times$ freq). The bisection bandwidth is a good metric because it relates the connectivity pattern and performance based on the achieved frequency.

A plot of the Cost Metric is shown in Figure 5. An interesting observation is that for 8-node systems the cost is practically the same, which is consistent with the results from the experiments in Section 5. For the 16-node systems, the star is the best topology because of its small diameter and easy implementation although the costs are still similar, except for the fully-connected system. As the number of nodes is increased to 32 , the star fails because it cannot meet timing due to congestion at the central node, and its cost becomes very high. The ring looks good at 32 nodes because of its low routing requirements but it has a high diameter. 
At 32 nodes, the cost of the ring starts to look worse than the mesh or hypercube because the diameter is increasing more rapidly. With respect to the hypercube, the mesh uses less logic and routing per node, but has a larger diameter so the two costs still remain similar. For larger chips that would allow even greater numbers of nodes, the trend suggests that the mesh and hypercube will continue to do well without modifying the capacity of the current FPGA routing fabric. These results are not surprising because when the limits of the routing capacity in the FPGA are reached, it is expected that the known properties of the various topologies would become more relevant.

If we were to consider a 256-node hypercube, the node degree is 8 , which is comparable to the the network interface for the 8-node fully-connected system, which can be routed. This suggests that the congestion around the nodes will not be a limiting factor for achieving complete routing and meeting timing. However, the link complexity for the 256-node hypercube is 1024 and it is only 28 for the 8-node fully-connected system. It is possible that the actual capacity of the routing channels will not support the 256-node system. Therefore, a higher capacity routing fabric will be required.

\section{CONCLUSIONS}

We have shown that for the same size of FPGA, using 64bit links (32 bits in each direction), the maximum difference between the 16-node ring, star, mesh and hypercube topologies is less than $8 \%$ of the total routing resources used and that they can all be mapped to run at about $180 \mathrm{MHz}$, the maximum speed of the processor. Even a fully-connected topology up to 16 nodes can be implemented but with a much higher overhead and a slight loss in performance.

The fully-connected topology fails to route at 32 nodes, with 56 nets unrouted, even though only about $50 \%$ of the logic is utilized. This system is at approximately the limits of the device routing capacity, meaning there are just not enough wires, independent of whether timing can be met, but there is room to implement more nodes. These results are reflected in our cost metric, which shows that at 16 nodes the more highly connected topologies start to fail, when timing considerations are also included. Up to 16 nodes, there is little need to worry about making tradeoffs using topologies with less connectivity. A 16-node NoC implemented on an FPGA using 64-bit buses should just use point-topoint connections. If the width of the link is increased, the expectation is that fewer nodes could be supported.

The 32-node fully-connected $\mathrm{NoC}$ is the limit of what the current routing fabric can support. The routing capacity of the device would have to be increased to achieve complete routing, and to meet timing, more of the faster types of routing tracks are required.

The generic topologies may provide greater system-level connectivity than required. In these situations applicationspecific topologies may be used that reduce connectivity, thus reducing the strain on the routing fabric's resources, allowing systems with larger numbers of nodes to be implemented.

\section{ACKNOWLEDGMENTS}

Thanks to Jorge Carrillo and Trevor Bauer at Xilinx for their feedback and helpful comments. We acknowledge Xilinx and CMC Microsystems/SOCRN for providing the tools, development hardware and computing equipment. Thanks to CONACYT for the funding provided to Manuel Saldaña and to the reviewers for their insightful comments.

\section{REFERENCES}

[1] ARM Corporation. "AMBA specification". [online] 1999. www.arm.com (Accessed: 2005).

[2] IBM Corporation. "The Coreconnect Bus Architecture", [online] 1999. www.chips.ibm.com (Accessed: 2005).

[3] OpenCores.org. "The WISHBONE System Architecture" [online] 2002. opencores.org/projects.cgi/web/wishbone (Accessed: 2005).

[4] Sonics Inc. (online). www.sonicsinc.com/sonics/products/siliconbackplaneIII/ (Accessed: 2005).

[5] G. de Micheli and L. Benini. Networks on chip: A new paradigm for systems on chip design. In DATE '02: Proceedings of the conference on Design, automation and test in Europe, page 418, Washington, DC, USA, 2002. IEEE Computer Society.

[6] S Kumar, A Jantsch, J Soininen, M Forsell, M Millberg, J Oberg, K Tiensyrja, and A Hemani. A network on chip architecture and design methodology. In VLSI, 2002. Proceedings. IEEE Computer Society Annual Symposium on, pages 105-112, Pittsburgh, USA, 2002. IEEE Computer Society.

[7] Adrijean Adriahantenaina, Herve Charlery, Alain Greiner, Laurent Mortiez, and Cesar Albenes Zeferino. Spin: A scalable, packet switched, on-chip micro-network. In DATE '03: Proceedings of the conference on Design, Automation and Test in Europe, page 20070, Washington, DC, USA, 2003 IEEE Computer Society.

[8] Partha Pratim Pande, Cristian Grecu, Michael Jones, André Ivanov, and Resve Saleh. Performance evaluation and design trade-offs for network-on-chip interconnect architectures. IEEE Trans. Comput., 54(8):1025-1040, 2005.

[9] William J. Dally and Brian Towles. Route packets, not wires: on-chip interconnection networks. In $D A C$ '01: Proceedings of the 38th conference on Design automation, pages 684-689, New York, NY, USA, 2001. ACM Press.

[10] G. Brebner and D. Levi. Networking on Chip with Platform FPGAs. In Field-Programmable Technology (FPT), Proceedings. 2003 IEEE International Conference on, pages 13-20, July 2003.

[11] Tim Kogel, Malte Doerper, Andreas Wieferink, Rainer Leupers, Gerd Ascheid, Heinrich Meyr, and Serge Goossens. A modular simulation framework for architectural exploration of on-chip interconnection networks. In CODES+ISSS '03: Proceedings of the 1st IEEE/ACM/IFIP international conference on Hardware/software codesign and system synthesis, pages 7-12, New York, NY, USA, 2003. ACM Press.

[12] Davide Bertozzi and Antoine Jalabert. NoC Synthesis Flow for Customized Domain Specific Multiprocessor Systems-on-Chip. IEEE Trans. Parallel Distrib. Syst., 16(2):113-129, 2005.

[13] Charles L. Seitz. Let's route packets instead of wires. In AUSCRYPT'90: Proceedings of the sixth MIT conference on Advanced research in VLSI, pages 133-138, Cambridge, MA, USA, 1990. MIT Press.

[14] T.A. Bartic, J.Y. Mignolet, T. Marescaux, D. Verkest, S Vernalde, and R. Lauwereins. Topology adaptive network-on-chip design and implementation. In Computer and Digital Tecniques, IEEE Proceedings, pages 467-472. IEE Proceedings, July 2005.

[15] J Duato and L Yalamanchili Ni. Interconnection Networks, an Engineering Approach. Computer Society Press, Los Alamitos, California, 1998.

[16] Xilinx, Inc. http://www.xilinx.com.

[17] ModelSim Home Page, [online] September 2005. http://www.model.com/. 